\title{
Advances in understanding genome maintenance
}

\author{
Kyle M Miller*
}

\begin{abstract}
A report from the Abcam genome stability conference 'Maintenance of Genome Stability', Jolly Beach Resort, Antigua, 8-11 March 2010.
\end{abstract}

'Maintenance of Genome Stability' is a biennial meeting that brings together, in a fantastic venue, diverse researchers working on how the integrity of genomes is maintained. Topics included DNA repair pathways, replication and recombination, and common themes included how these processes are regulated during the cell cycle, in the context of their chromatin or genomic location, and their involvement in cancer. Here, I summarize some highlights of the meeting, which included large-scale genomic studies, work on post-translational modifications in genome maintenance, and insights into new mechanisms and proteins involved in DNA repair pathways, telomeres and cancer.

\section{Mass spectrometry proteomics}

Post-translational modifications in DNA damage signaling was a common theme throughout the meeting. Jiri Bartek (Centre for Genotoxic Stress Research, Copenhagen, Denmark) presented a genome-wide proteomics screen, using quantitative mass spectrometry (stable isotope labeling with amino acids in cell culture, SILAC), for phosphorylations of nuclear proteins that change following DNA damage in human cells. Over 7,000 phosphorylation sites were detected, with 2,000 being novel and not described in other proteomic screens. The power of this screen was that it analyzed the temporal regulation of phosphorylations after DNA damage. To accomplish this, cells were analyzed at time zero (no damage) and then at 5 minutes, 20 minutes, 1 hour and 8 hours after DNA damage. Taking into account only those phosphorylations detected at all time points, almost 600 phosphorylations were found to change by

*Correspondence: k.miller@gurdon.cam.ac.uk

The Gurdon Institute and Department of Biochemistry, University of Cambridge, Tennis Court Road, Cambridge CB2 1QN, UK over twofold. Induced phosphorylations and dephosphorylations were detected. Phosphorylations that increased at early time points after DNA damage were enriched in substrates for the kinase ataxia-telangiectasia mutated (ATM). Looking at the amino acid sequences surrounding the phosphorylation sites, a new phosphorylation consensus sequence, SxxQ, was determined that does not seem to be mediated by any known kinase. This dataset is very likely to be a useful resource for researchers interested in phosphorylation in the DNA damage response (DDR).

Post-translational modifications other than phosphorylation are also important for the DDR, and it is clear that ubiquitylation has a key role in DNA damage signaling. Work from Jiri Lukas' group (Center for Genotoxic Stress Research, Copenhagen, Denmark) and others have added strong evidence for this notion by identifying two ubiquitin E3 ligases, RNF8 and RNF168, as important regulators of DNA damage signaling. To gain insights into the substrates of these two enzymes, Lukas' group performed quantitative mass spectrometry (SILAC) of 6xHis-Flag-Ub-containing complexes from cells lacking RNF8 or RNF168. Many DDR proteins were found to be ubiquitylated in an RNF8- or RNF168-dependent manner. Of the histones, only histone $\mathrm{H} 2 \mathrm{~A}$ and histone H2AX were purified and verified as substrates. Therefore, ubiquitin not only decorates the chromatin around a double-strand break (DSB) but also is conjugated to many signaling proteins, which again highlights the importance of this modification in the DDR. An understanding of how ubiquitylation affects DNA repair and signaling proteins to bring about proper repair is guaranteed to be a continued topic of research.

Chromatin is an important component in promoting genomic and epigenomic stability. Anja Groth (BRIC, University of Copenhagen, Denmark) discussed recent findings from her group showing the histone modifications on histones $\mathrm{H} 3$ and $\mathrm{H} 4$ that are associated with the histone chaperone Asf1b in S phase or in response to replication impaired by hydroxyurea (HU). Complexes of Asf1b containing histones $\mathrm{H} 3$ and $\mathrm{H} 4$ were purified and quantified by mass spectrometry. Groth described how all Asf1b-bound $\mathrm{H} 4$ molecules were acetylated on Lys5 and Lys12; histone $\mathrm{H} 3$ was 30\% acetylated on Lys18 and $20 \%$ acetylated on Lys14; and H3 was also 
mono-methylated at Lys9. Acetylation of H3 on Lys56 was barely detectable, suggesting that this mark is not a general mark of histones in $\mathrm{S}$ phase. During replication stress (HU), methylation of $\mathrm{H} 3$ on Lys9 (H3K9me1) increased significantly in Asf1b complexes. Thus, this epigenetic mark is deregulated after replication stress, and Asf1b seems to have a role in sequestering $\mathrm{H} 3$ molecules containing this mark. As H3K9me1 can affect epigenetic silencing, this work suggests that replication stress could affect epigenomic integrity through Asf1b.

\section{Site-specific DNA double-strand breaks}

The DNA damage field has long awaited the arrival of a robust system for studying site-specific DSBs in mammalian cells. This wait might be over because two research groups revealed such systems. Gaelle Legube (University of Toulouse, France) showed a new site-specific DSB system that uses a rare cutting restriction enzyme that is regulatable. The break sites are found throughout the genome and can be analyzed simultaneously. Chromatin immunoprecipitation microarray (ChIP-chip) analysis demonstrated robust phospho-histone H2AX $(\gamma \mathrm{H} 2 \mathrm{AX})$ formation at many predicted genomic break sites. Legube found that large $\gamma \mathrm{H} 2 \mathrm{AX}$ domains formed bidirectionally from the DNA breaks but were not uniform or symmetrical, suggesting genomic boundaries that limit $\gamma \mathrm{H} 2 \mathrm{AX}$ spreading. Active transcription still occurred in $\gamma \mathrm{H} 2 \mathrm{AX}$ domains, although the level of $\gamma \mathrm{H} 2 \mathrm{AX}$ was reduced around transcription start sites, possibly because of increased histone dynamics.

Roger Greenberg (University of Pennsylvania, Philadelphia, USA) presented data from a system that uses an integrated Lac operator ( $\mathrm{LacO})$ array that is positioned approximately $4 \mathrm{~kb}$ upstream of a transcriptionally active site, which can be monitored in real time for DSB responses and nascent transcript formation. In this system, a LacI-FokI nuclease fusion protein is expressed that site-specifically binds the LacO array and creates site-specific damage. This is a great tool to look at the effects of DNA damage on transcription. Indeed, Greenberg's group found that, following DNA damage, transcription was rapidly halted, with a concomitant compaction of the genomic region due to DSB-induced inhibition of RNA polymerase II elongation. These effects were all dependent on the DDR kinase ATM. The transcriptional silencing occurred in cis, as creation of DNA damage outside this region did not affect transcription from the reporter locus. Silencing was rapidly reversible following DSB repair, as were all the effects described. Finally, ubiquitylated histone $\mathrm{H} 2 \mathrm{~A}$ was detected at this region and its deubiquitylation by the ubiquitin-specific peptidase 16 (USP16) was required for resumption of transcription following DNA repair. Thus, DNA damage within multiple kilobases of a transcriptionally active locus results in transcriptional repression that is dependent on ATM and ubiquitin.

\section{Novel proteins and processes in genome maintenance \\ Deubiquitylation in the DNA damage response}

As ubiquitin conjugation has been linked with DNA damage surveillance and repair, systems must be present to modulate or counteract these pathways. Daniel Durocher's group (Samuel Lunenfeld Research Institute, Toronto, Canada) has pioneered the use of genome-wide screens using small interfering RNA (siRNA) to look microscopically at how knockdown of individual genes affects DNA damage signaling and protein localization at DNA damage sites. Durocher's group previously identified two ubiquitin E3 ligase enzymes, RNF8 and RNF168, that are required for DNA repair through their combined ubiquitylation of substrates, including histones, at sites of DNA damage. Durocher's group has now performed a siRNA screen targeting the human deubiquitylating enzymes (DUBs) to analyze how these enzymes can affect ubiquitin conjugates at DNA damage sites. Daniel Durocher presented in a talk his groups findings from this screen that revealed several candidate genes whose knockdown resulted in the accumulation of ubiquitin conjugates at unrepaired DNA damage. Thus, these data show that systems do indeed exist that function to deubiquitylate substrates at sites of DNA damage. Defining the DUBs and their key targets that function in the DDR, as well as determining how ubiquitin affects these pathways, are important questions in the field. As the ubiquitin pathway is extremely complex, answers to these questions will undoubtedly uncover many surprises and interesting biology that will be important in advancing our understanding of the role of ubiquitin in genome maintenance.

\section{Fanconi anemia}

Fanconi anemia (FA) is a rare genetic disorder resulting in an increased incidence of some cancers and heightened cellular sensitivity to inter-strand DNA cross-linking agents. John Rouse (University of Dundee, UK) and Josef Jiricny (University of Zurich, Switzerland) introduced a new FA-associated protein, REND1/KIAA1018, which functions as a 5' flap endonuclease and an exonuclease in the repair of inter-strand crosslinks. Following DNA damage, this protein forms foci by binding the ubiquitinconjugated form of Fanconi anemia group D2 protein (FANCD2), revealing that it functions downstream of FANCD2. Loss of REND1/KIAA1018 resulted in hypersensitivity to inter-strand DNA crosslinks, showing that it is a bona fide participant of the FA pathway. Whether mutations in REND1/KIAA1018 are found in human patients suffering from Fanconi anemia is yet to be 
determined but this work suggests that there may be additional, as-yet-unidentified proteins that function in this complex repair pathway, which is essential for maintaining genome stability.

\section{Telomeres and cancer}

Aneuploidy is prominent in many cancers and it has been hypothesized that tetraploidy initializes these events. Dysfunctional telomeres are also a common initiating event in cancer but a link between these two processes has not yet been established. Titia de Lange (Rockefeller University, New York, USA) presented data showing how telomere dysfunction can cause endoreduplication, resulting in tetraploidy in p53-deficient cells. Deprotection of telomeres by the absence of the telomereassociated Pot1a/b proteins resulted in cell cycle arrest, which was dependent on the protein ataxia-telangiectasia and Rad3-related (ATR), and tetraploidy. Using a powerful microscopy technique, fluorescence ubiquitination cell cycle indicator (FUCCI), de Lange showed in vivo data of these cells degrading geminin and re-expressing the origin licensing factor $\mathrm{Cdt} 1$ in the absence of mitosis, resulting in endoreduplication. When telomere endprotection was reintroduced, these tetraploid cells could resume growth. This mechanism of tetraploidization is not limited to telomere deprotection because DNA damage can result in the same outcome. Thus, these data reveal how telomeres and DNA damage can result in tetraploidy, a cellular state seen in cancer and implicated in tumorigenesis.

This successful conference series has become a mustattend meeting in the field of genome stability. Although details are unavailable for 2012, this meeting is sure to supply great science to people who are interested in the mechanisms that govern genome integrity.

\section{Acknowledgements}

I thank the Wellcome Trust for providing my funding to attend this meeting.

Published: 20 April 2010

doi:10.1186/gb-2010-11-4-301

Cite this article as: Miller KM: Advances in understanding genome maintenance. Genome Biology 2010, 11:301. 